\title{
Construction and analysis of a IncRNA-miRNA-mRNA network based on competitive endogenous RNA reveals functional IncRNAs in diabetic cardiomyopathy
}

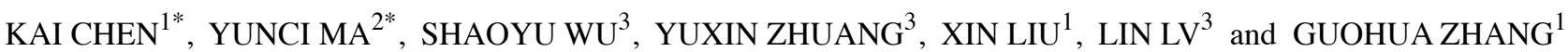 \\ ${ }^{1}$ School of Traditional Chinese Medicine, Southern Medical University, Guangzhou, Guangdong 510515; \\ ${ }^{2}$ Nanfang Hospital, Southern Medical University/The First School of Clinical Medicine, \\ Southern Medical University, Guangzhou, Guangdong 510000; ${ }^{3}$ School of Pharmaceutical Sciences, \\ Southern Medical University, Guangzhou, Guangdong 510515, P.R. China
}

Received July 12, 2018; Accepted February 19, 2019

DOI: $10.3892 / \mathrm{mmr} .2019 .10361$

\begin{abstract}
Diabetic cardiomyopathy (DCM) is a major cause of mortality in patients with diabetes, particularly those with type 2 diabetes. Long non-coding RNAs (lncRNAs), including terminal differentiation-induced lncRNA (TINCR), myocardial infarction-associated transcript (MIAT) and H19, serve a key role in the regulation of DCM. MicroRNAs (miRNAs/miRs) can inhibit the expression of mRNA at the post-transcriptional level, whereas lncRNAs can mask the inhibitory effects of miRNAs on mRNA. Together, miRNAs and lncRNAs form a competitive endogenous non-coding RNA (ceRNA) network that regulates the occurrence and development of various diseases. However, the regulatory role of lncRNAs in DCM is unclear. In this study, a background network containing mRNAs, miRNAs and lncRNAs was constructed using starBase and a regulatory network of DCM was screened using Cytoscape. A functional lncRNA, $\mathrm{X}$-inactive specific transcript (XIST), was identified in the disease network and the main miRNAs (miR-424-5p and miR-497-5p) that are regulated by XIST were further screened
\end{abstract}

Correspondence to: Mr. Guohua Zhang, School of Traditional Chinese Medicine, Southern Medical University, 1838 North Guangzhou Avenue, Guangzhou, Guangdong 510515, P.R. China E-mail: zghgz@163.com

Miss Lin Lv, School of Pharmaceutical Sciences, Southern Medical University, 1838 North Guangzhou Avenue, Guangzhou, Guangdong 510515, P.R. China

E-mail: lynnlv@smu.edu.cn

"Contributed equally

Abbreviations: DCM, diabetic cardiomyopathy; AGEs, advanced glycation end products; DEGs, differentially expressed genes; GIFtS, GeneCards Inferred Functionality Scores; HF, heart failure

Key words: diabetes, diabetic cardiomyopathy, competitive endogenous RNA, long non-coding RNA, microRNA, gene chip, bioinformatics analysis to obtain the ceRNA regulatory network of DCM. In conclusion, the results of this study revealed that lncRNAs may serve an important role in DCM and provided novel insights into the pathogenesis of DCM.

\section{Introduction}

Diabetic cardiomyopathy (DCM) is a type cardiomyopathy caused by diabetes mellitus; the main pathological alterations are cardiac hypertrophy and cardiac dysfunction (1). Clinical studies have reported that DCM is the main reason underlying the high incidence and high mortality rate of heart failure (HF) in patients with diabetes $(2,3)$. However, the mechanism underlying DCM is not entirely clear; therefore, there is no effective targeted therapy (4). Research has increasingly focused on identifying the mechanism underlying DCM and exploring potential effective treatments.

Non-coding RNAs (ncRNAs) are functional RNA molecules in the transcriptome that do not encode proteins, including microRNAs (miRNAs/miRs) and long ncRNAs (lncRNAs) (5). miRNAs are single-stranded, non-coding small RNAs that are highly conserved in evolution and have post-transcriptional regulatory activity (6), whereas lncRNAs are mRNA-like transcripts $>200$ nucleotides long that have no or little protein-coding function and serve important roles in numerous biological processes (7). IncRNAs have been reported to be important in the governing of fundamental biological processes (8), and their aberrant expression may be associated with the pathogenesis of various diseases, including cancer, neurodegenerative diseases, and cardiovascular diseases (9).

Previous studies have demonstrated that lncRNAs can act as competitive endogenous RNAs (ceRNAs) to compete with target genes for miRNA response elements and attenuate the inhibitory effect of miRNAs on target genes $(10,11)$. Therefore, they can indirectly regulate the expression of target genes and affect the occurrence and development of diseases (12), particularly cardiovascular disease (13). Zhang et al (14), reported that the lncRNA metastasis-associated lung adenocarcinoma transcript 1 serves a role in the pathogenesis of DCM, and 
Zhou et al (15) demonstrated that lncRNA myocardial infarction-associated transcript is a ceRNA that upregulates death-associated protein kinase 2 by inhibiting miR-22-3p in DCM. However, the association between ceRNAs and DCM is unclear. Therefore, the present study aimed to elucidate the pathogenesis of DCM from the perspective of ceRNA using bioinformatics analysis. The pipeline of construction and analysis of the IncRNA-miRNA-mRNA network based on ceRNA in DCM is shown in Fig. 1.

\section{Materials and methods}

Identification of DCM-related genes. DCM-related genes were collected from two main sources: Gene Expression Omnibus (GEO) and GeneCards. The GEO (https://www.ncbi. nlm.nih.gov/gds/) is a public functional genomics data repository supporting minimum information about a microarray experiment-compliant data submissions. For the present study, the GSE26887 dataset was obtained, which includes DCM and normal myocardial tissue gene expression profiles (16). The GSE26887 dataset includes microarray data from seven patients with type 2 diabetes mellitus (T2DM) and $\mathrm{HF}$, and five control individuals. Subsequently, GeoDiver (https://www.geodiver.co.uk/) (17) was used to develop overview boxplots, a volcano plot, and a heat map to show the distribution of differentially expressed genes (DEGs). $\mathrm{P}<0.01$ and $[\log$ fold change (FC)] $>1.5$ were considered statistically significant.

GeneCards is a database for searchable human gene annotations (http://www.genecards.org/). Its gene-centric data are automatically mined from $\sim 125$ web sources, including genomic, transcriptomic, proteomic, genetic, clinical and functional information. After entering a keyword of 'diabetic cardiomyopathy' into GeneCards, relevance score $>30$ and GeneCards Inferred Functionality Scores (18) $>60$ were used as cut-off criteria to collect disease-related genes and merge them with DEGs.

miRNA-mRNA and lncRNA-miRNA interactions. The ceRNA theory dictates that the construction of a ceRNA background network (CBGN) requires large numbers of lncRNAs, miRNAs, mRNAs and their interactions. Firstly, miRNA-mRNA and IncRNA-miRNA interactions were obtained from the starBase V2.0 database (http://starbase.sysu.edu.cn/). StarBase was designed to decode miRNA-mRNA, miRNA-ceRNA, miRNA-lncRNA, miRNA-circRNA, miRNA-pseudogene and protein-RNA interaction networks from cross-linking immunoprecipitation-sequencing data.

Construction of the ceRNA background network. Cytoscape software v3.5.1 (http://www.cytoscape.org/) was used to integrate and combine miRNA-mRNA and IncRNA-miRNA interactions to construct the lncRNA-miRNA-mRNA (ceRNA) background network. Cytoscape is an open-source software platform used to visualize complex networks and integrate these with any type of attribute data. In Cytoscape, IncRNAs, miRNAs and mRNAs are expressed in different nodes, which are connected by lines to indicate an interaction between them. Finally, an intricate network diagram consisting of lncRNAs, miRNAs and mRNAs was obtained.
Table I. Differentially expressed genes identified from the GSE26887 dataset using GeoDiver.

\begin{tabular}{lll}
\hline Gene symbol & logFC & P-value \\
\hline NPPA & -3.53 & $1.36 \times 10^{-10}$ \\
SFRP4 & -2.71 & $2.96 \times 10^{-5}$ \\
DSC1 & -2.44 & $1.35 \times 10^{-6}$ \\
NEB & -2.40 & $6.83 \times 10^{-5}$ \\
FRZB & -2.36 & $7.56 \times 10^{-6}$ \\
AK125574 & -2.09 & $5.16 \times 10^{-6}$ \\
ENPP2 & -2.01 & $4.50 \times 10^{-5}$ \\
PRELP & -1.90 & $2.18 \times 10^{-5}$ \\
NPR3 & -1.87 & $1.12 \times 10^{-5}$ \\
IGSF10 & -1.72 & $6.26 \times 10^{-6}$ \\
HSPA2 & -1.71 & $5.91 \times 10^{-5}$ \\
COL14A1 & -1.67 & $2.44 \times 10^{-5}$ \\
P2RY14 & -1.62 & $1.27 \times 10^{-5}$ \\
SLC9B1 & -1.57 & $1.56 \times 10^{-5}$ \\
FMOD & -1.56 & $3.42 \times 10^{-5}$ \\
SVEP1 & -1.53 & $5.54 \times 10^{-6}$ \\
FAXDC2 & -1.52 & $1.77 \times 10^{-5}$ \\
CNN1 & 1.57 & $4.55 \times 10^{-5}$ \\
GFPT2 & 1.61 & $9.96 \times 10^{-5}$ \\
CD163 & 1.67 & $1.35 \times 10^{-6}$ \\
KCNIP2 & 1.90 & $8.40 \times 10^{-5}$ \\
S100A8 & 1.98 & $2.68 \times 10^{-5}$ \\
ANKRD2 & 2.16 & $1.89 \times 10^{-6}$ \\
& & \\
& &
\end{tabular}

FC, fold change.

Signaling pathway enrichment analysis. Signaling pathway enrichment of DCM-related genes was conducted using the Cytoscape plugin ClueGO (http://apps.cytoscape. org/apps/cluego) (19). ClueGO is a Cytoscape plugin that visualizes non-redundant biological terms for large clusters of genes in a functionally grouped network; it is created with kappa statistics and reflects the relationships between the terms based on the similarity of their associated genes. For this study, Kyoto Encyclopedia of Genes and Genomes (KEGG) analysis (20) was selected in ClueGO to obtain information about the signaling pathways regulated by DCM-related genes.

Construction of a DCM-related ceRNA network (DCMCN). The DCMCN was extracted from CBGN using the network-merge tool in Cytoscape. DCM-related genes and CBGN were imported into Cytoscape together to construct two separate networks. The two networks were then merged using the network merge operation (21) of Cytoscape to build a new network. The new network comprised DCM-related genes and relevant miRNAs and lncRNAs; this network is known as the DCMCN.

Analysis of the DCMCN. Hub nodes serve critical roles in gene networks (22). Therefore, all node degrees of the IncRNA-miRNA-mRNA network were calculated. Specifically, the network-analyzer tool (23) in Cytoscape was 

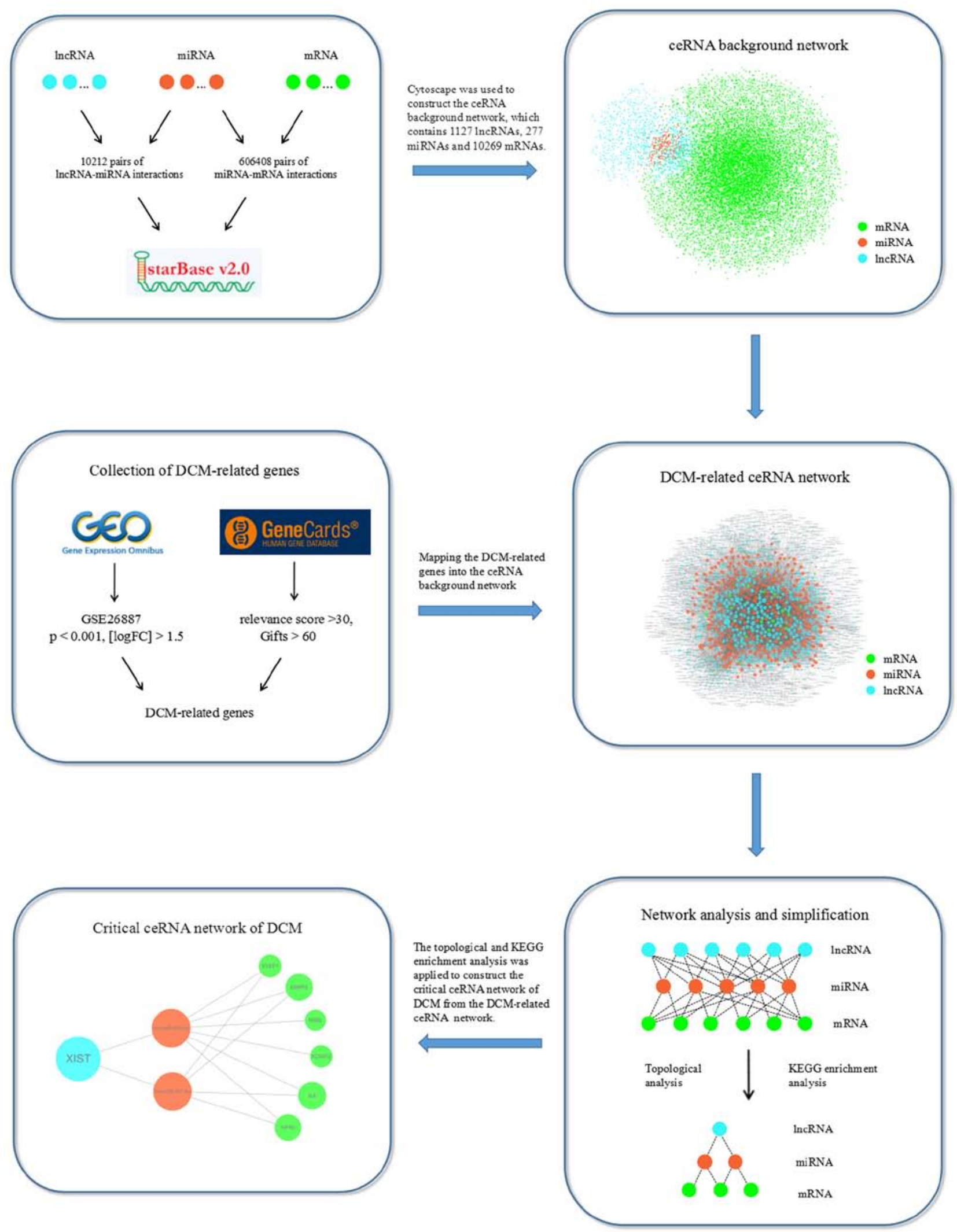

Figure 1. Integrative flowchart for the construction of the ceRNA background network, DCM-related ceRNA network, and critical ceRNA network of DCM.

used to obtain the topological parameters of the network, mainly the degree value. Degree is the most direct measure of centrality in network analysis. Generally, the higher the degree of a node, the more critical it is to the network (24). Finally, the IncRNA with the largest degree from the network was selected, which likely serves a key role in regulating DCM. Subsequently, the IncRNA and its downstream miRNAs and mRNAs were selected in Cytoscape and isolated from the DCMCN to form a new network. The miRNAs bound by the IncRNA were screened synthetically for target sites in 


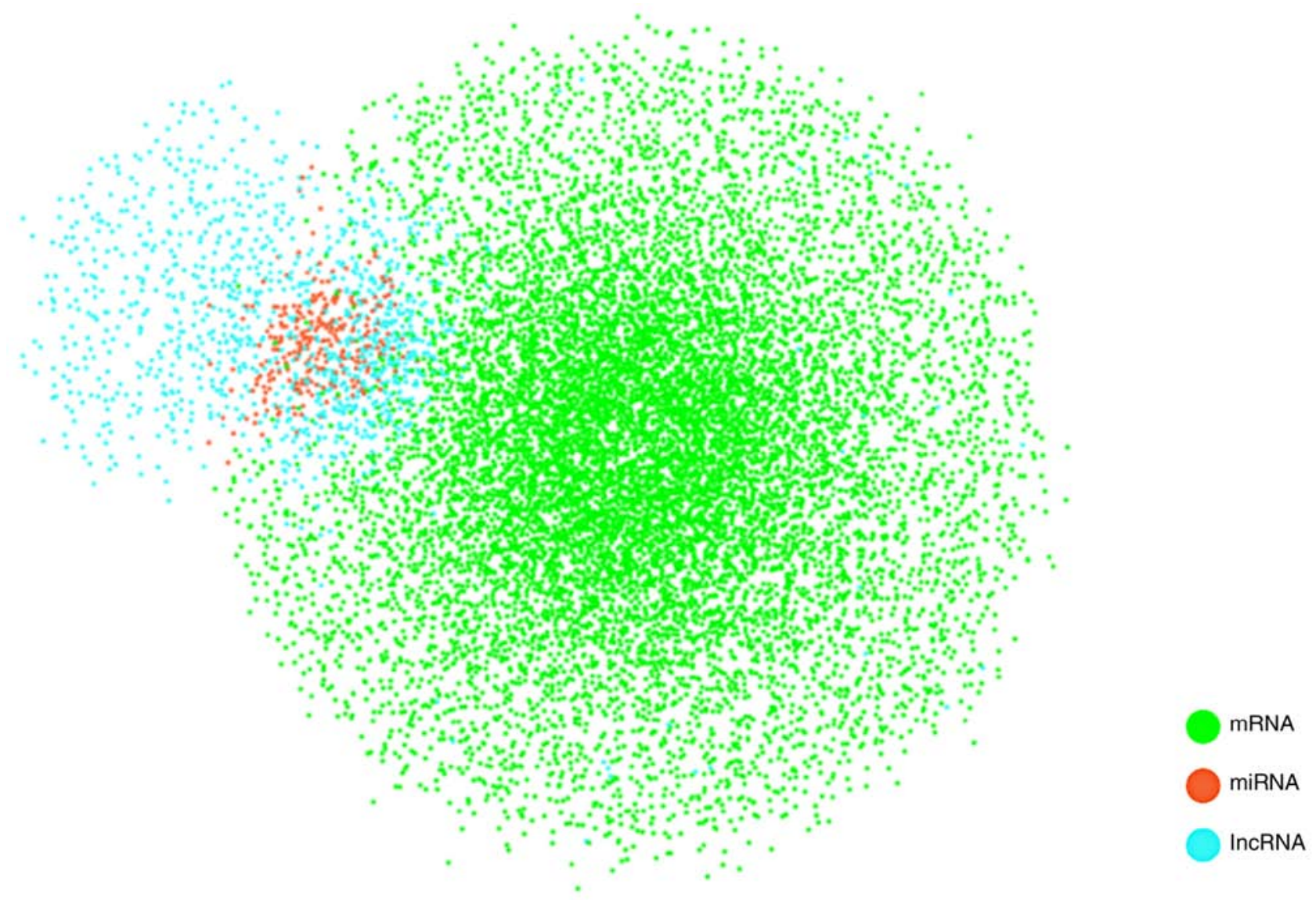

Figure 2. View of the competing endogenous RNA background network. The blue nodes represent lncRNAs, the orange nodes represent miRNAs and the green nodes represent mRNAs. There were 1,127 lncRNAs, 277 miRNAs, and 10,269 mRNAs in the network. IncRNA, long non-coding RNA; miRNA, microRNA.

starBase, and degree and number of target genes in Cytoscape. The major miRNAs and their target genes regulated by the lncRNA were obtained and plotted into a small ceRNA regulatory network using Cytoscape. Finally, the ClueGO plugin in Cytoscape was used to analyze the gene function of each regulatory network to determine whether it was consistent with the regulatory function of the DCMCN.

\section{Results}

Construction of the ceRNA background network. To construct the ceRNA background network, the miRNA-mRNA and IncRNA-miRNA interactions were downloaded from starBase v2.0. The data in starBase v2.0 are mainly collected from TargetScan (http://www.targetscan.org/vert_71/) (25), RNA22 (https://cm.jefferson.edu/rna22/) (26), picTar (http://pictar.mdc-berlin.de/) (27), PITA (http://genie.weizmann.ac.il/pubs/mir07/mir07_data.html) and miRanda (http://www.microrna.org/microrna/home.do?) (28). A total of 606,408 pairs of miRNA-mRNA interactions and 10,212 pairs of IncRNA-miRNA interactions were obtained and Cytoscape software v3.5.1 was then used to integrate the miRNA-mRNA and IncRNA-miRNA information to construct the IncRNA-miRNA-mRNA (ceRNA) background network (Fig. 2).

Identification of DCM-related genes. DCM-related genes were collected from the GEO and GeneCards. Firstly, the open gene expression profile GSE26887 was downloaded the from GEO database. Subsequently, GeoDiver was used to analyze the DEGs between controls and patients with T2DM and HF in the GSE26887 dataset. An overview boxplot, volcano plot and heatmap were generated using GeoDiver (Fig. 3A-C), and DEGs were identified.

As shown in Table I, 23 DEGs were identified from the GEO repository microarray data using cutoff criteria of $\mathrm{P}<0.01$ and $[\log \mathrm{FC}]>1.5$. Subsequently, GeneCards databases were searched for disease-related genes (Table II). After removing redundancy, 53 genes were identified as DCM-related genes, including NPPA, SFRP4, DSC1, NEB, FRZB, AK125574, ENPP2, PRELP, NPR3, IGSF10, HSPA2, COL14A1, P2RY14, SLC9B1, FMOD, SVEP1, FAXDC2, CNN1, GFPT2, CD163, KCNIP2, S100A8, ANKRD2, ACE, F2, IL6, NOS3, TNF, AGTR1, MTHFR, REN, APOB, APOE, LMNA, TNNI3, TGFB1, NPPB, INS, APOA1, FBN1, CRP, F5, THBD, CST3, EDN1, SCN5A, GP1BA, PON1, ACTC1, DES, PTPN11, CCL2, TNNT2.

Signaling pathwayenrichment analysisfor DCM-related genes. DCM-related genes were examined through ClueGO-mediated enrichment analysis by employing KEGG terms for the annotation of gene function. As shown in Fig. 4A and B, DCM-related genes were mainly associated with the 'AGE-RAGE signaling pathway in diabetic complications'.

Construction and analysis of the DCMCN from CBGN. To observe regulation of ceRNAs in DCM and identify DCM-related 


\section{B}

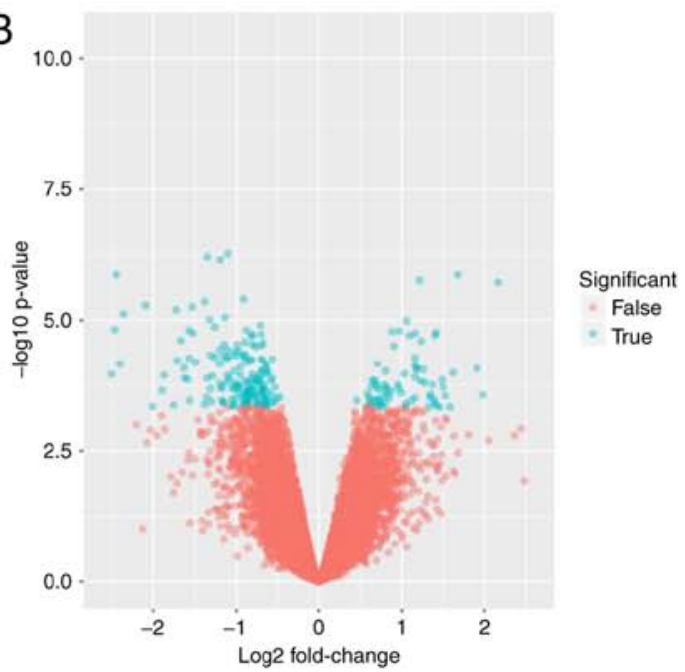

\section{C}

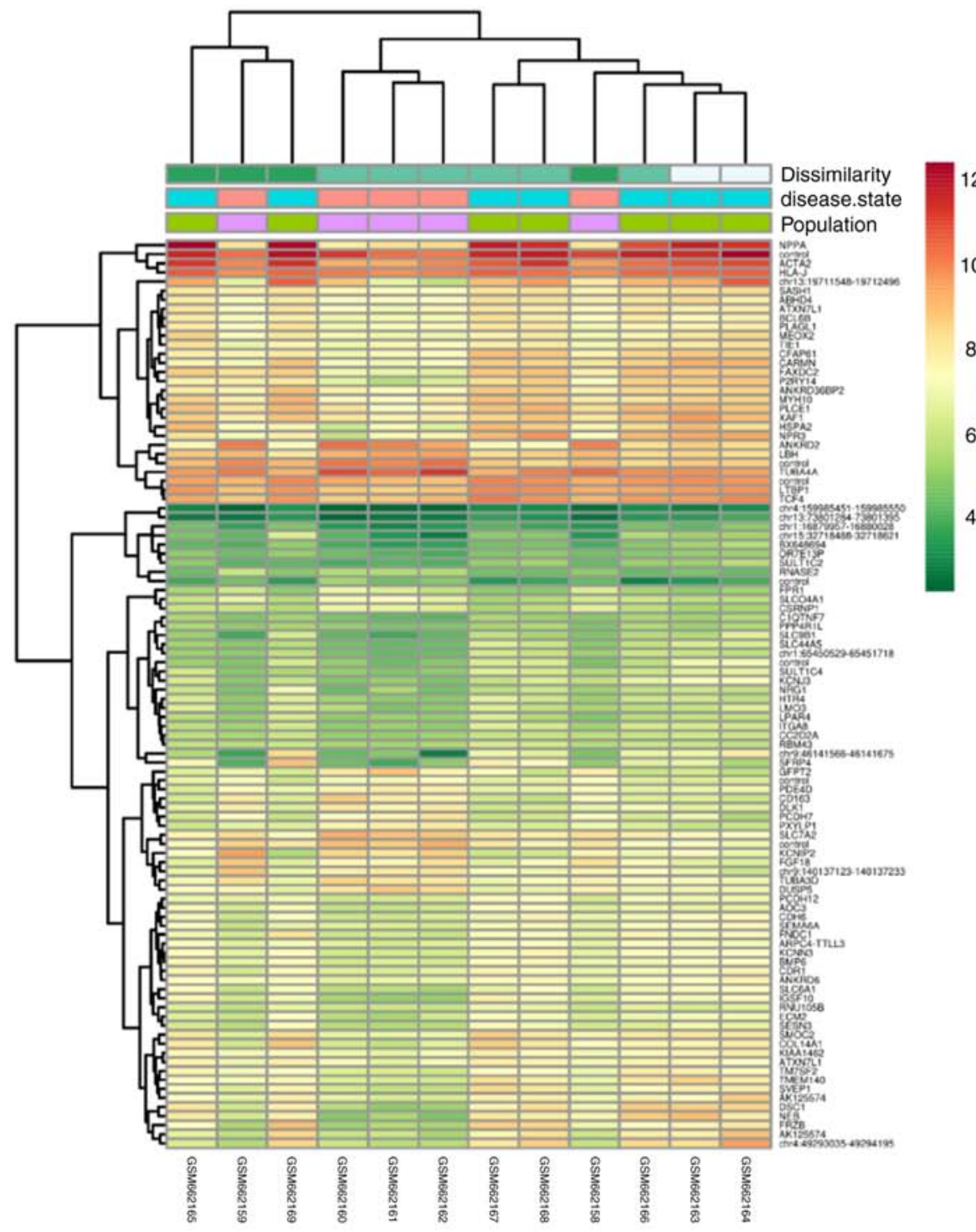

Figure 3. Identification of differentially expressed genes from the GSE26887 dataset using GeoDiver. (A) Overview boxplot, (B) volcano plot and (C) heatmap were generated using GeoDiver. Group A are controls, whereas Group B are patients with type 2 diabetes mellitus and heart failure.

lncRNAs, 53 DCM-related genes (mRNAs) were mapped into the ceRNA background network. As shown in Fig. 5A, the DCMCN was extracted from the ceRNA background network using the network merge tool in Cytoscape. The blue, orange and green nodes represent lncRNAs, miRNAs and DCM-related genes, respectively. In addition, the edges represent the interactions 
Table II. Disease-related genes collected from GeneCards.

\begin{tabular}{|c|c|c|c|}
\hline Gene symbol & Gene name & GIFtS & Relevance score \\
\hline ACE & Angiotensin I converting enzyme & 70 & 82.65 \\
\hline F2 & Coagulation factor II, thrombin & 70 & 55.55 \\
\hline IL6 & Interleukin 6 & 70 & 54.18 \\
\hline NOS3 & Nitric oxide synthase 3 & 72 & 52.43 \\
\hline TNF & Tumor necrosis factor & 77 & 51.78 \\
\hline AGTR1 & Angiotensin II receptor type 1 & 71 & 51.43 \\
\hline MTHFR & Methylenetetrahydrofolate reductase & 64 & 50.99 \\
\hline REN & Renin & 68 & 49.63 \\
\hline APOB & Apolipoprotein B & 65 & 47.05 \\
\hline APOE & Apolipoprotein E & 71 & 46.92 \\
\hline LMNA & Lamin $\mathrm{A} / \mathrm{C}$ & 66 & 42.33 \\
\hline TNNI3 & Troponin I3, cardiac type & 69 & 42.21 \\
\hline TGFB1 & Transforming growth factor $\beta 1$ & 75 & 42.09 \\
\hline NPPB & Natriuretic peptide B & 63 & 41.2 \\
\hline INS & Insulin & 67 & 39.05 \\
\hline NPPA & Natriuretic peptide A & 65 & 36.24 \\
\hline APOA1 & Apolipoprotein A1 & 70 & 35.38 \\
\hline FBN1 & Fibrillin 1 & 62 & 34.86 \\
\hline CRP & $\mathrm{C}$-reactive protein & 68 & 34.74 \\
\hline F5 & Coagulation factor $\mathrm{V}$ & 63 & 34.44 \\
\hline THBD & Thrombomodulin & 62 & 33.6 \\
\hline CST3 & Cystatin C & 64 & 33.38 \\
\hline EDN1 & Endothelin 1 & 67 & 32.87 \\
\hline SCN5A & Sodium voltage-gated channel $\alpha$ subunit 5 & 70 & 32.8 \\
\hline GP1BA & Glycoprotein Ib platelet alpha Subunit & 63 & 31.03 \\
\hline PON1 & Paraoxonase 1 & 65 & 30.99 \\
\hline ACTC1 & Actin, $\alpha$, cardiac muscle 1 & 60 & 30.96 \\
\hline DES & Desmin & 68 & 30.88 \\
\hline PTPN11 & Protein tyrosine phosphatase, non-receptor type 11 & 71 & 30.71 \\
\hline CCL2 & $\mathrm{C}-\mathrm{C}$ motif chemokine ligand 2 & 70 & 30.67 \\
\hline TNNT2 & Troponin T2, cardiac type & 67 & 30.2 \\
\hline
\end{tabular}

between lncRNAs, miRNAs and mRNAs. The network analyzer tool in Cytoscape was used to analyze the topological parameters of the network; the top 10 degrees are shown in Table III. Notably, lncRNA X-inactive specific transcript (XIST) had the largest degree in the DCMCN, suggesting that lncRNA-XIST may be a key lncRNA that regulates the development of DCM.

KEGG pathway analysis was performed for lncRNA-XIST. IncRNA-XIST and its downstream miRNAs and mRNAs were selected in Cytoscape and isolated from the DCMCN to reconstruct a new ceRNA network (Fig. 5B). To simplify the ceRNA XIST network, the number of target sites, degrees, and target genes of the miRNAs regulated by XIST were further screened (Table IV). Information regarding the target sites of the miRNAs was obtained from starBase. After screening, six miRNAs (hsa-miR-424-5p, hsa-miR-497-5p, hsa-miR-16-5p, hsa-miR-15b-5p, hsa-miR-15a-5p and hsa-miR-195-5p) had the maximum parameters of target sites, degrees and target genes.
Table III. Degree value of lncRNAs in the diabetic cardiomyopathy-related ceRNA network.

\begin{tabular}{lr}
\hline lncRNA & Degree \\
\hline XIST & 175 \\
CTA-204B4.6 & 130 \\
MALAT1 & 99 \\
ZNF518A & 83 \\
KCNQ1OT1 & 75 \\
OIP5-AS1 & 71 \\
NEAT1 & 69 \\
DCP1A & 69 \\
HCG18 & 64 \\
FGD5-AS1 & 62 \\
\hline
\end{tabular}


A
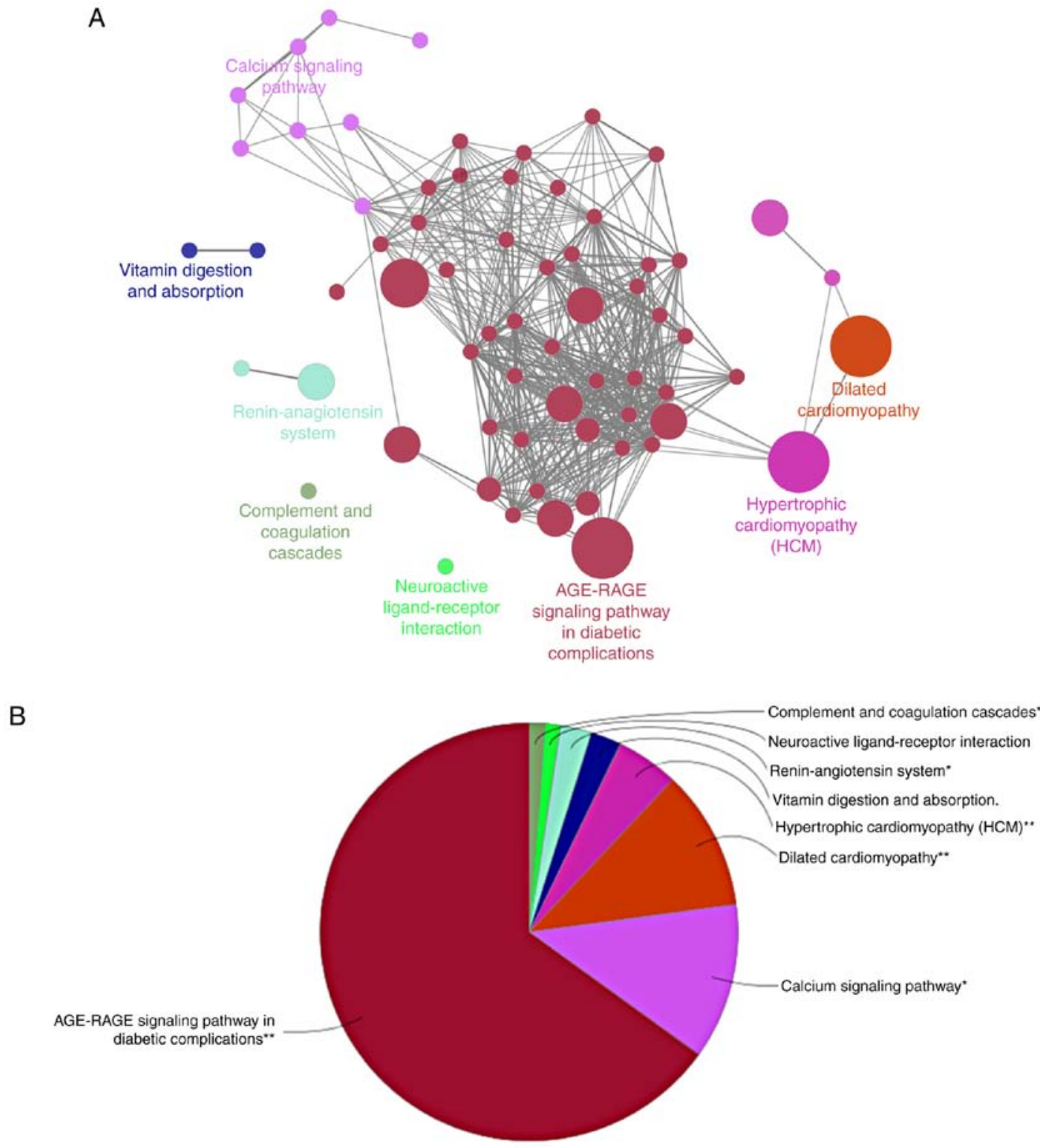

Figure 4. Pathway analysis of DCM-related genes using ClueGO. (A) A functionally grouped network and (B) pie chart of enriched categories was generated for the target genes. Kyoto Encyclopedia of Genes and Genomes terms are represented as nodes, and node size represents the enrichment significance of the term. Functionally related groups partially overlap. Only the significant terms in the group were labeled: ${ }^{*} \mathrm{P}<0.05$ and ${ }^{* *} \mathrm{P}<0.01$.

These six miRNAs and their target genes that were regulated by the IncRNA were obtained and plotted into a small ceRNA network using Cytoscape software (Fig. 6A). KEGG enrichment analysis demonstrated that only hsa-miR-424-5p and hsa-miR-497-5p may act on the 'AGE-RAGE signaling pathway in diabetic complications' (Fig. 6B and C).

\section{Discussion}

In the present study, a comprehensive bioinformatics approach was used to examine key lncRNAs involved in DCM and to elucidate their molecular mechanisms in the development of DCM. In particular, interaction data from starBase were used to generate a ceRNA background network based on the theory of ceRNA. Subsequently, a DCMCN was extracted from the ceRNA background network by mapping the DCM-related genes. In total, the DCMCN contained 24 mRNA nodes, 320 miRNA nodes, 1,127 lncRNA nodes, and 10,808 edges.
Table IV. Target sites, degree, and number of target genes of the miRNAs bound by $\mathrm{X}$-inactive specific transcript.

\begin{tabular}{lccc}
\hline Name & Target sites & Degree & Target genes \\
\hline hsa-miR-424-5p & 5 & 152 & 6 \\
hsa-miR-497-5p & 5 & 150 & 4 \\
hsa-miR-16-5p & 5 & 150 & 4 \\
hsa-miR-15b-5p & 5 & 150 & 4 \\
hsa-miR-15a-5p & 5 & 149 & 3 \\
hsa-miR-195-5p & 5 & 149 & 3 \\
hsa-miR-485-5p & 1 & 78 & 3 \\
hsa-miR-20a-5p & 4 & 77 & 3 \\
hsa-miR-20b-5p & 4 & 76 & 3 \\
hsa-miR-93-5p & 4 & 77 & 3 \\
\hline
\end{tabular}

miRNA/miR, microRNA. 


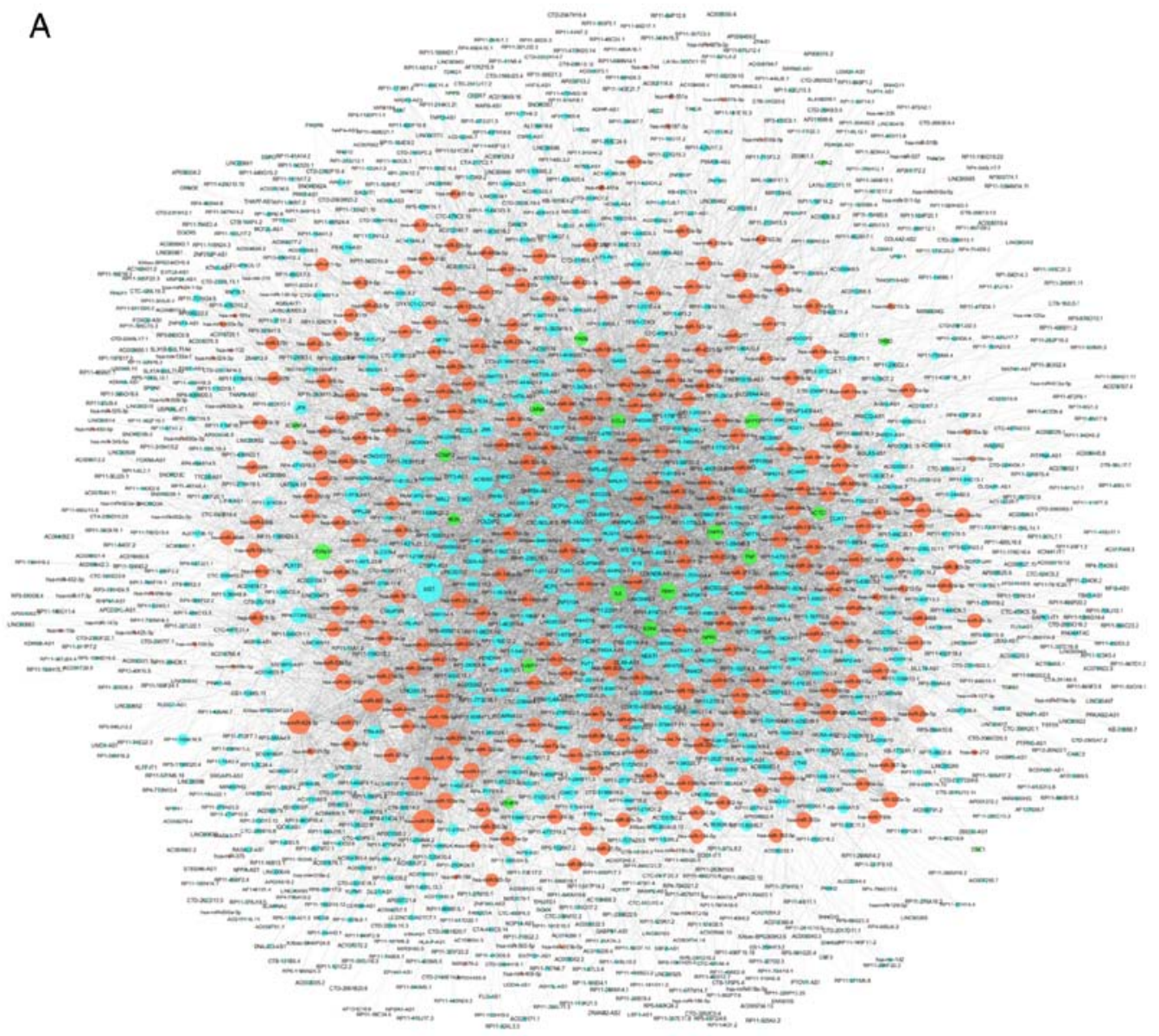

B

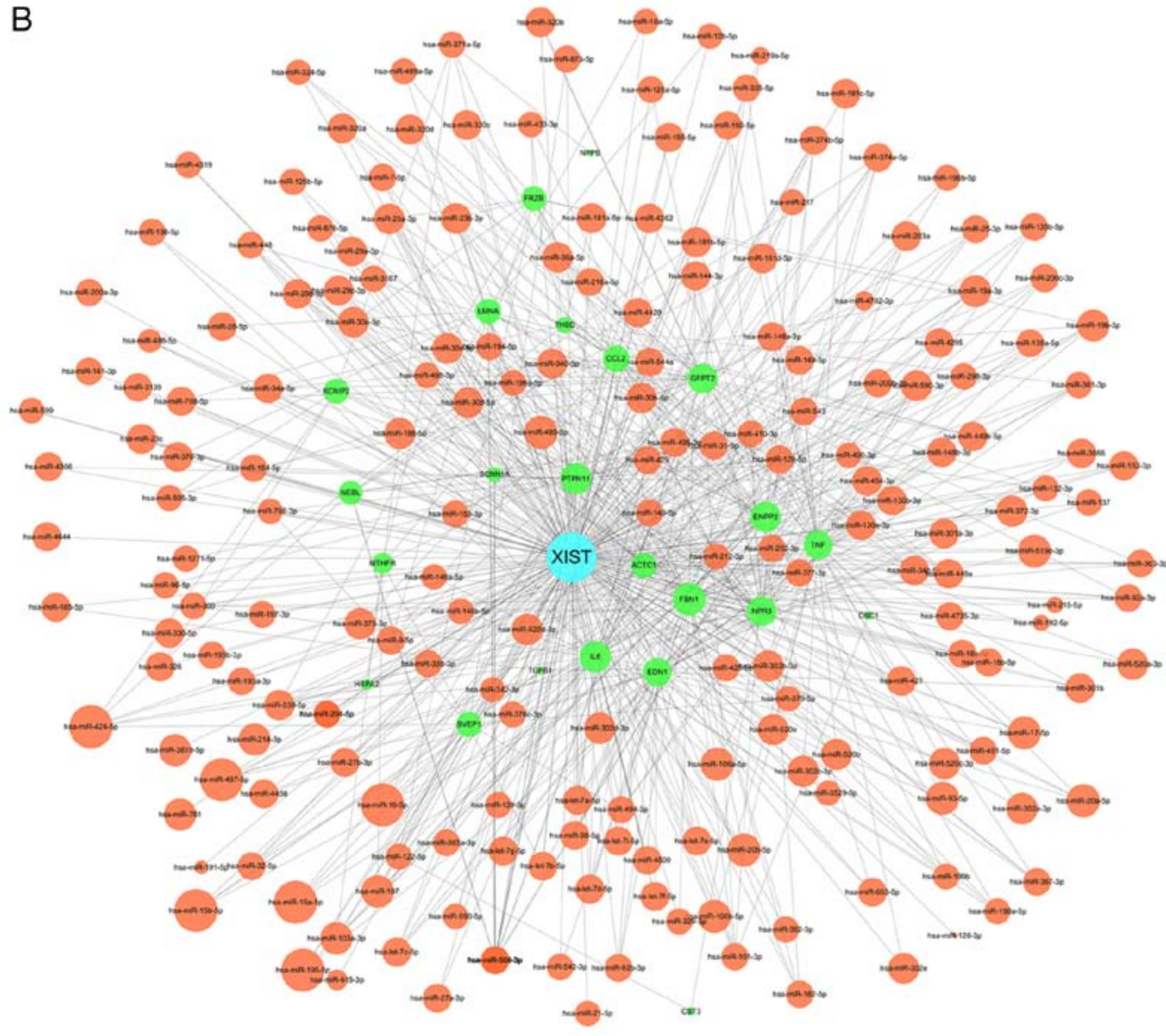

mRNA
miRNA
IncRNA

Figure 5. Construction and analysis of a DCMCN using Cytoscape. (A) DCMCN. (B) XIST-associated ceRNA network. The blue nodes represent lncRNAs, the orange nodes represent miRNAs and the green nodes represent miRNAs. ceRNA, competing endogenous RNA; DCMCN, diabetic cardiomyopathy-related ceRNA network; lncRNA, long non-coding RNA; miRNA, microRNA; XIST, X-inactive specific transcript. 
A

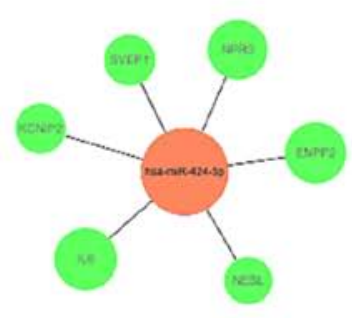

hsa-miR-424-5p

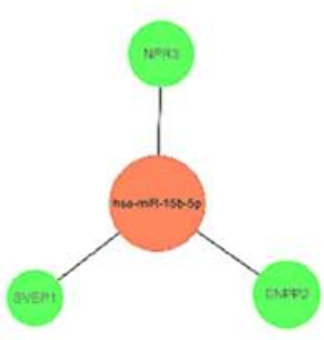

hsa-miR-15b-5p

B

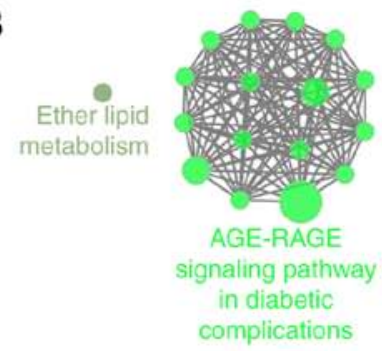

hsa-miR-424-5p

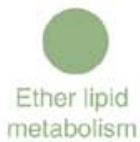

hsa-miR-15b-5p

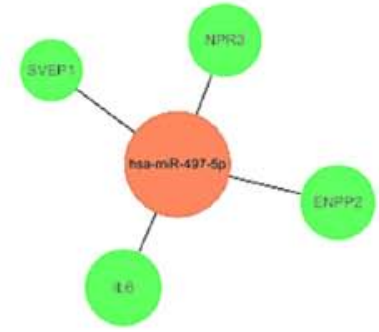

hsa-miR-497-5p

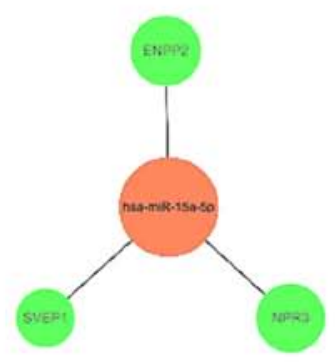

hsa-miR-15a-5p

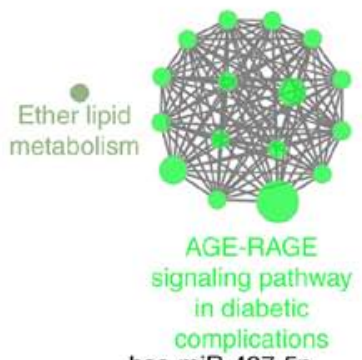

hsa-miR-497-5p

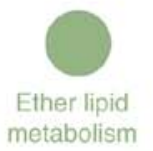

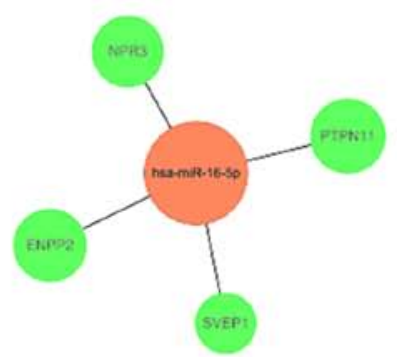

hsa-miR-16-5p

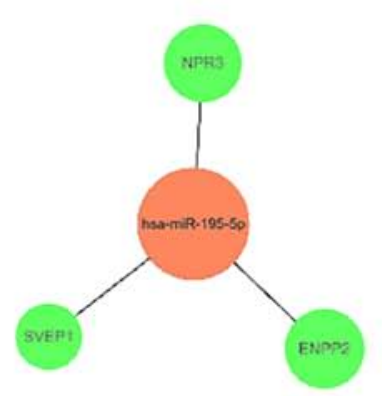

hsa-miR-195-5p

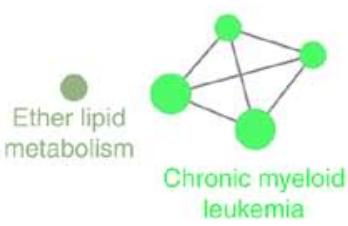

hsa-miR-16-5p

Ether lipid metabolism

\section{C}

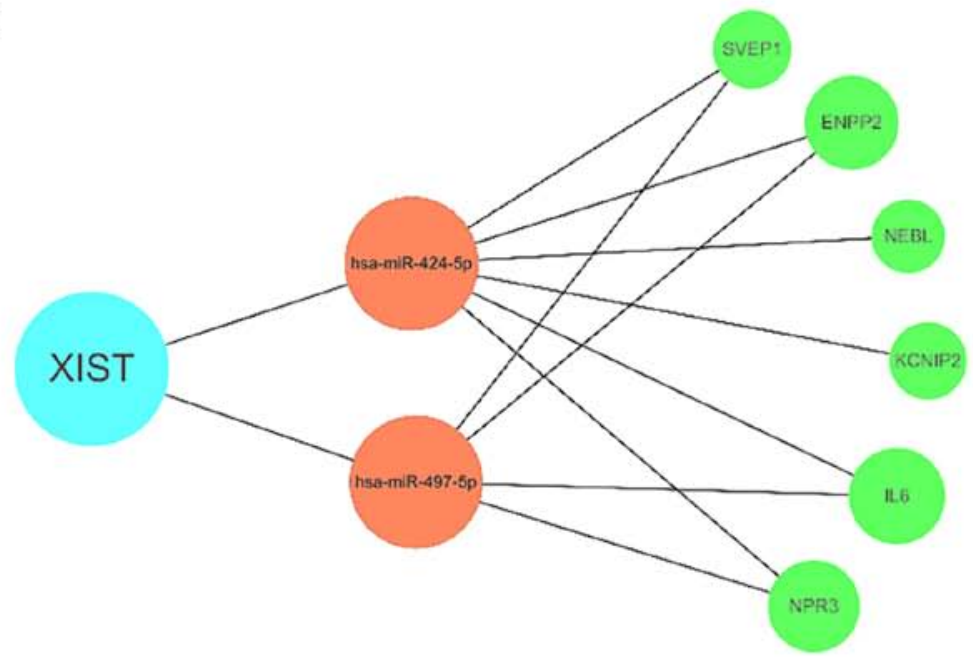

Figure 6. ceRNA regulatory network of the miRNAs bound by XIST and the gene function of each network. (A) ceRNA regulatory network of the pivotal miRNAs bound by XIST. (B) A functionally grouped network of enriched categories was generated for the target genes of the pivotal miRNAs bound by XIST. Kyoto Encyclopedia of Genes and Genomes terms are represented as nodes, and node size represents the enrichment significance of the term. (C) ceRNA regulatory network of XIST in diabetic cardiomyopathy following optimization with ClueGO. ceRNA, competing endogenous RNA; miR/miRNA, microRNA; XIST, X-inactive specific transcript. 
Subsequently, topological properties were assessed and a cluster analysis was performed on the DCMCN.

The results revealed that the lncRNA XIST could directly interact with several miRNAs with known relevance to the development of DCM. Comprehensive analysis revealed that the downstream targets of XIST, hsa-miR-424-5p and hsa-miR-497-5p, may be pivotal miRNAs that regulate DCM.

Hsa-miR-424-5p is located at the $\mathrm{X}$ chromosome, and is potentially among the $15 \%$ of X-linked genes that escape female X-chromosome inactivation (XCI), resulting in higher expression in women (29). A previous study demonstrated that hsa-miR-424-5p is significantly downregulated in peripheral blood from patients with HF, thus suggesting that it may be considered a potential biomarker and contributor to HF (30). In addition, upregulation of miR-424-5p promotes downstream processes associated with hypoxia, including angiogenesis and erythropoiesis, to ameliorate myocardial ischemia, which is considered a cardioprotective factor (31).

Hsa-miR-497-5p is located on chromosome 17 and is highly conserved in several species (32). This miRNA is a member of the miR-15/107 group that includes the seed sequence AGCAGC, which is an important determinant of target recognition (33). It has previously been reported that miR-497-5p is closely associated with cardiac fibrosis through activation of latent transforming growth factor (TGF)- $\beta 1$ anchored in the extracellular matrix by targeting the 3'-untranslated region of reversion-inducing cysteine rich protein with Kazal motifs (34). In addition, a previous study revealed the negative regulatory effect of hsa-miR-497-5p against SMAD family member 3 transcripts, which suggests the possible role of this miRNA in regulation of the TGF $\beta$ signaling pathway (35).

XIST is a lncRNA (17 kb in Homo sapiens) required for $\mathrm{XCI}$ of one of the two $\mathrm{X}$ chromosomes in female cells, thus enabling dosage compensation between $\mathrm{XX}$ females and XY males (36). XCI takes place early in embryonic development, and is thought to occur in multiple steps: Counting and choosing the $\mathrm{X}$ chromosome to silence, spreading of XIST over the target $\mathrm{X}$ chromosome, and silencing most of its active genes (37). In recent years, IncRNA XIST has been reported to serve a role as a regulatory factor of tumor proliferation. It has been demonstrated that XIST has an important positive role in pancreatic cancer proliferation (38), colorectal cancer (39) and other types of cancer by targeting corresponding miRNAs. Furthermore, IncRNA XIST has been reported to suppress the proliferation of myocardial cells and promote apoptosis by targeting miR-130a-3p in myocardial infarction (40). However, to the best of our knowledge, there is currently no evidence regarding the regulatory role of lncRNA XIST in DCM.

To the best of our knowledge, this study is the first to identify a ceRNA network including lncRNA XIST, hsa-miR-424-5p, hsa-miR-497-5p and DCM-related genes in DCM. In addition, KEGG analysis revealed that the 'AGE-RAGE signaling pathway in diabetic complications' may be a major pathway leading to the development of DCM.

The accumulation of advanced glycation end products (AGEs) has a crucial role in the onset and progress of diabetic nephropathy (41). AGEs interact with the receptor for AGEs (RAGE) on the cell membrane and induce deleterious effects via activation of nuclear factor $\kappa \mathrm{B}$, ultimately leading to increased vascular permeability and inflammation (42). RAGE is formed from the cleavage of the native membrane receptor mediated by disintegrins and matrix metalloproteinases (43), and circulates in the blood. It has previously been reported that AGE-RAGE interaction in diabetes can negatively affect endothelial cell physiology, resulting in increased predisposition toward cardiovascular disease (44); this has been reported in clinical studies wherein patients with diabetes exhibit higher RAGE expression (45). Animal models of diabetic atherosclerosis have also demonstrated improved regression of atherosclerotic plaques following RAGE knockout (46). Therefore, understanding the AGE-RAGE axis in the development of endothelial dysfunction and its regulation by lncRNAs may be helpful in designing novel therapies that target endothelial dysfunction and impair development of cardiovascular diseases.

In conclusion, the present study constructed a background network containing mRNAs, miRNAs and lncRNAs, and then a regulatory network of DCM was screened. Finally, a functional lncRNA XIST was identified in the network and the main miRNAs (miR-424-5p and miR-497-5p) that are regulated by XIST were further screened to obtain the ceRNA regulatory network of DCM. The present study highlighted the involvement of IncRNA XIST in DCM, and facilitated the development of lncRNA-directed diagnostic and therapeutic tools against diabetes mellitus. These findings improved our knowledge on the mechanism of DCM and may provide potential therapeutic target in the treatment of DCM in clinic.

\section{Acknowledgements}

Not applicable.

\section{Funding}

The present study was supported by the National Nature Science Foundation of China (grant nos. 81072937 and 81473246).

\section{Availability of data and materials}

The datasets used and/or analyzed during the current study are available from the corresponding author on reasonable request.

\section{Authors' contributions}

KC, YM, LL and GZ conceived and designed the experiments. $\mathrm{KC}$ and SW performed the experiments. YM and YZ analyzed the data. KC and XL contributed reagents, materials and analytical tools and contributed to the analysis and interpretation of data.. All authors read and approved the final manuscript.

\section{Ethics approval and consent to participate}

Not applicable.

\section{Patient consent for publication}

Not applicable.

\section{Competing interests}

The authors declare that they have no competing interests. 


\section{References}

1. Jia G, Whaley-Connell A and Sowers JR: Diabetic cardiomyopathy: A hyperglycaemia-and insulin-resistance-induced heart disease. Diabetologia 61: 21-28, 2018.

2. Seferović PM and Paulus WJ: Clinical diabetic cardiomyopathy: A two-faced disease with restrictive and dilated phenotypes. Eur Heart J 36: 1718-1727, 2015.

3. Lam CS: Diabetic cardiomyopathy: An expression of stage B heart failure with preserved ejection fraction. Diab Vasc Dis Res 12: 234-238, 2015.

4. Aneja A, Tang WH, Bansilal S, Garcia MJ and Farkouh ME: Diabetic cardiomyopathy: Insights into pathogenesis, diagnostic challenges, and therapeutic options. Am J Med 121: 748-757, 2008.

5. Taft RJ, Pang KC, Mercer TR, Dinger M and Mattick JS: Non-coding RNAs: Regulators of disease. J Pathol 220: 126-139, 2010.

6. Iwakawa $\mathrm{H}$ and Tomari Y: The functions of MicroRNAs: MRNA decay and translational repression. Trends Cell Biol 25: 651-665, 2015 .

7. Jandura A and Krause HM: The New RNA World: Growing Evidence for long noncoding RNA functionality. Trends Genet 33: 665-676, 2017.

8. Li W, Notani D and Rosenfeld MG: Enhancers as non-coding RNA transcription units: Recent insights and future perspectives. Nat Rev Genet 17: 207-223, 2016.

9. Thum T and Condorelli G: Long Noncoding RNAs and MicroRNAs in cardiovascular pathophysiology. Circ Res 116: 751-762, 2015.

10. Salmena L, Poliseno L, Tay Y, Kats L and Pandolfi PP: A ceRNA Hypothesis: The rosetta stone of a hidden RNA language? Cell 146: 353-358, 2011

11. Smillie CL, Sirey T and Ponting CP: Complexities of post-transcriptional regulation and the modeling of ceRNA crosstalk. Crit Rev Biochem Mol Biol 53: 231-245, 2018

12. Shi X, Sun M, Liu H, Yao Y and Song Y: Long non-coding RNAs: A new frontier in the study of human diseases. Cancer Lett 339: 159-166, 2013

13. Dong Y, Liu C, Zhao Y, Ponnusamy M, Li P and Wang K: Role of noncoding RNAs in regulation of cardiac cell death and cardiovascular diseases. Cell Mol Life Sci 75: 291-300, 2018.

14. Zhang $\mathrm{M}, \mathrm{Gu} \mathrm{H}$, Chen $\mathrm{J}$ and Zhou $\mathrm{X}$ : Involvement of long noncoding RNA MALAT1 in the pathogenesis of diabetic cardiomyopathy. Int J Cardiol 202: 753-755, 2016.

15. Zhou X, Zhang W, Jin M, Chen J, Xu W and Kong X: lncRNA MIAT functions as a competing endogenous RNA to upregulate DAPK2 by sponging miR-22-3p in diabetic cardiomyopathy. Cell Death Dis 8: e2929, 2017

16. Greco S, Fasanaro P, Castelvecchio S, D'Alessandra Y, Arcelli D, Di Donato M, Malavazos A, Capogrossi MC, Menicanti L and Martelli F: MicroRNA dysregulation in diabetic ischemic heart failure patients. Diabetes 61: 1633-1641, 2012.

17. Ismail M, Suresh H, Nazrath N, Anisatu R, Marian P, Bruno V, Fabrizio S and Conrad B: GeoDiver: Differential gene expression analysis \& gene-set analysis for GEO Datasets. Queen Mary J Intellectual Property: Apr 15, 2017 doi: https://doi. org/10.1101/127753.

18. Harel A, Inger A, Stelzer G, Strichman-Almashanu L, Dalah I, Safran M and Lancet D: GIFtS: Annotation landscape analysis with GeneCards. BMC Bioinformatics 10: 348, 2009.

19. Bindea G, Mlecnik B, Hackl H, Charoentong P, Tosolini M, Kirilovsky A, Fridman WH, Pagès F, Trajanoski $\mathrm{Z}$ and Galon J: ClueGO: A Cytoscape plug-in to decipher functionally grouped gene ontology and pathway annotation networks. Bioinformatics 25: 1091-1093, 2009.

20. Kanehisa M, Furumichi M, Tanabe M, Sato Y and Morishima K KEGG: New perspectives on genomes, pathways, diseases and drugs. Nucleic Acids Res 45: D353-D361, 2017.

21. Su G, Morris JH, Demchak B and Bader GD: Biological network exploration with Cytoscape 3. Curr Protoc Bioinformatics 47 $1-24,2014$

22. Han JD, Bertin N, Hao T, Goldberg DS, Berriz GF, Zhang LV, Dupuy D, Walhout AJ, Cusick ME, Roth FP and Vidal M: Evidence for dynamically organized modularity in the yeast protein-protein interaction network. Nature 430: 88-93, 2004

23. Cline MS, Smoot M, Cerami E, Kuchinsky A, Landys N, Workman C, Christmas R, Avila-Campilo I, Creech M, Gross B, et al: Integration of biological networks and gene expression data using Cytoscape. Nat Protoc 2: 2366-2382, 2007

24. Zhou B, Wang B and Zhe H: Degree-layer theory of network topology. Phys Soc: 18 Sep, 2014.
25. Fromm B, Billipp T, Peck LE, Johansen M, Tarver JE, King BL, Newcomb JM, Sempere LF, Flatmark K, Hovig E and Peterson KJ: A Uniform system for the annotation of vertebrate microRNA genes and the evolution of the human microRNAome. Annu Rev Genet 49: 213-242, 2015.

26. Miranda KC, Huynh T, Tay Y, Ang YS, Tam WL, Thomson AM, Lim B and Rigoutsos I: A pattern-based method for the identification of MicroRNA binding sites and their corresponding heteroduplexes. Cell 126: 1203-1217, 2006.

27. Krek A, Grün D, Poy MN, Wolf R, Rosenberg L, Epstein EJ, MacMenamin P, da Piedade I, Gunsalus KC Stoffel M and Rajewsky N: Combinatorial microRNA target predictions. Nature Genet 37: 495-500, 2005.

28. Betel D, Koppal A, Agius P, Sander C and Leslie C: Comprehensive modeling of microRNA targets predicts functional non-conserved and non-canonical sites. Genome Biol 11: R90, 2010.

29. Carrel L and Willard HF: X-inactivation profile reveals extensive variability in X-linked gene expression in females. Nature 434: 400-404, 2005

30. Marques FZ, Vizi D, Khammy O, Mariani JA and Kaye DM: The transcardiac gradient of cardio-microRNAs in the failing heart. Eur J Heart Fail 18: 1000-1008, 2016.

31. Ghosh G, Subramanian IV, Adhikari N, Zhang X, Joshi HP, Basi D, Chandrashekhar YS, Hall JL, Roy S, Zeng Y and Ramakrishnan S: Hypoxia-induced microRNA-424 expression in human endothelial cells regulates HIF- $\alpha$ isoforms and promotes angiogenesis. J Clin Invest 120: 4141-4154, 2010.

32. Flavin RJ, Smyth PC, Laios A, O'Toole SA, Barrett C, Finn SP, Russell S, Ring M, Denning KM, Li J, et al: Potentially important microRNA cluster on chromosome $17 \mathrm{p} 13.1$ in primary peritoneal carcinoma. Mod Pathol 22: 197-205, 2009.

33. Finnerty JR, Wang WX, Hébert SS, Wilfred BR, Mao G and Nelson PT: The miR-15/107 group of microRNA genes: Evolutionary biology, cellular functions, and roles in human diseases. J Mol Biol 402: 491-509, 2010.

34. Chen X, Shi C, Wang C, Liu W, Chu Y, Xiang Z, Hu K, Dong P and Han X: The role of miR-497-5p in myofibroblast differentiation of LR-MSCs and pulmonary fibrogenesis. Sci Rep 7: 40958, 2017.

35. Jafarzadeh M, Soltani BM, Dokanehiifard S, Kay M, Aghdami N and Hosseinkhani S: Experimental evidences for hsa-miR-497-5p as a negative regulator of SMAD3 gene expression. Gene 586: 216-221, 2016.

36. Gendrel AV and Heard E: Fifty years of X-inactivation research. Development 138: 5049-5055, 2011.

37. Payer B and Lee JT: X chromosome dosage compensation: How mammals keep the balance. Annu Rev Genet 42: 733-772, 2008.

38. Wei W, Liu Y, Lu Y, Yang B and Tang L: LncRNA XIST promotes pancreatic cancer proliferation through miR-133a/EGFR. J Cell Biochem 118: 3349-3358, 2017.

39. Song H, He P, Shao T, Li Y, Li J and Zhang Y: Long non-coding RNA XIST functions as an oncogene in human colorectal cancer by targeting miR-132-3p. J BUON 22: 696-703, 2017.

40. Zhou T, Qin G, Yang L, Xiang D and Li S: LncRNA XIST regulates myocardial infarction by targeting miR-130a-3p. J Cell Physiol 234: 8659-8667, 2019

41. Uribarri J, Cai W, Ramdas M, Goodman S, Pyzik R, Chen X, Zhu L, Striker GE and Vlassara H: Restriction of advanced glycation end products improves insulin resistance in human type 2 diabetes: Potential role of AGER1 and SIRT1. Diabetes Care 34: 1610-1616, 2011

42. Prasad K: Low levels of serum soluble receptors for advanced glycation end products, biomarkers for disease state: Myth or Reality. Int J Angiol 23: 11-16, 2014.

43. Tam XH, Shiu SW, Leng L, Bucala R, Betteridge DJ and Tan KC: Enhanced expression of receptor for advanced glycation end-products is associated with low circulating soluble isoforms of the receptor in Type 2 diabetes. Clin Sci (Lond) 120: 81-89, 2011.

44. Gao X, Zhang H, Schmidt AM and Zhang C: AGE/RAGE produces endothelial dysfunction in coronary arterioles in type 2 diabetic mice. Am J Physiol Heart Circ Physiol 295: H491-H498, 2008.

45. Villegas-Rodríguez ME, Uribarri J, Solorio-Meza SE, Fajardo-Araujo ME, Cai W, Torres-Graciano S, Rangel-Salazar R, Wrobel K and Garay-Sevilla ME: The AGE-RAGE axis and its relationship to markers of cardiovascular disease in newly diagnosed diabetic patients. PLoS One 11: e0159175, 2016

46. Soro-Paavonen A, Watson AM, Li J, Paavonen K, Koitka A, Calkin AC, Barit D, Coughlan MT, Drew BG, Lancaster GI, et al: Receptor for advanced glycation end products (RAGE) deficiency attenuates the development of atherosclerosis in diabetes. Diabetes 57: 2461-2469, 2008. 